九州大学学術情報リポジトリ

Kyushu University Institutional Repository

\title{
A MONOTONE FUZZY STOPPING TIME IN DYNAMIC FUZZY SYSTEMS
}

Yoshida, Yuji

Faculty of Economics and Business Administration, Kitakyushu University

Yasuda, Masami

Faculty of Science, Chiba University

Nakagami, Jun-ichi

Faculty of Science, Chiba University

Kurano, Masami

Faculty of Education, Chiba University

https://doi.org/10.5109/13482

出版情報: Bulletin of informatics and cybernetics. 31 (1), pp.91-99, 1999-03. Research Association of Statistical Sciences

バージョン :

権利関係 : 


\title{
A MONOTONE FUZZY STOPPING TIME IN DYNAMIC FUZZY SYSTEMS
}

\author{
By \\ Yuji Yoshida $^{\star}$, Masami Yasuda ${ }^{\dagger}$, Jun-ichi NaKagami ${ }^{\dagger}$ and Masami Kurano ${ }^{\ddagger}$
}

\begin{abstract}
This paper is concerned with a fuzzy stopping time for a dynamic fuzzy system. A new class of fuzzy stopping times which is called as a monotone fuzzy stopping time is introduced. The notion of monotonicity is well-known and important in the stochastic optimization theory. Here we will try to define a monotone property and discuss a stopping problem which is corresponding a dynamic fuzzy system. Since the fuzzy stopping time can be constructed using by $\alpha$-cuts of fuzzy states, the explicit derivation of an optimal one is derived under appropriate assumptions. The key point of our discussion for the optimization of a stopping problem is to induce an additive weighting function for the fuzzy reward.
\end{abstract}

\section{Introduction and notations}

The stopping time with fuzziness, called a 'fuzzy stopping time', is discussed in our previous paper, Kurano et al.(1998). The corresponding optimization problem on fuzzy stoppings is considered by the constructive method. This kind notion of stopping times are first introduced by Kacprzyk(1977) as time horizon constraints in the dynamic programming, and Kacprzyk and Esogbue(1996) studied multistage decision-making with fuzzy time constraints.

We have extended the problem to that of an optimization handling about a system with fuzzy states, which is called a 'dynamic fuzzy system' (Kurano et al.(1992), Yoshida(1994)). In Kurano et al.(1998), we also discussed a problem of systems in conjunction with fuzzy stopping times as strategies.

Alternatively, it is well-known that a class of stopping times with a monotone property is useful for various application problems concerning about stochastic models, because the class is simple to understand and easy to calculate. Refer to Chow, Robbins and Siegmund(1971), Ross(1970) etc..

In this paper, we introduce a new class of fuzzy stopping times which has a monotone property in a sense of fuzziness and apply it to a problem with additive weighting functions, which is incorporated into the scalarization of the fuzzy total rewards.

\footnotetext{
* Faculty of Economics and Business Administration, Kitakyushu University, 4-2-1 Kitagata, Kokuraminami, Kitakyushu 802-8577, Japan

† Faculty of Science, Chiba University, 1-33 Yayo-cho, Inage-ku, Chiba 263-8522, Japan

‡ Faculty of Education, Chiba University, 1-33 Yayo-cho, Inage-ku, Chiba 263-8522, Japan
} 
In the remainder of this section, a fuzzy dynamic system is described. In Section 2, a new class of fuzzy stopping times, which we call them 'monotone fuzzy stopping times', is introduced and their construction are also discussed. These results are applied to the 'optimization' of a corresponding fuzzy stopping problem in Section 3. In Section 4 , an example is given to illustrate the results.

Throughout the paper, we will denote a fuzzy set and a fuzzy relation by their membership functions defined on a convex compact subsets of some Banach space. For the theory of fuzzy sets, refer to Novák(1985). The detail of its definition is omitted here.

Let $E$ be a given convex compact subsets of some Banach space. $\mathcal{F}(E)$ denotes the set of all convex fuzzy sets, $\tilde{u}$, on $E$ whose membership functions are assumed to be upper semi-continuous and have a compact support with the normality condition: $\sup _{x \in E} \tilde{u}(x)=1$. The $\alpha$-cut $(\alpha \in[0,1])$ of the fuzzy set $\tilde{u}$ is denoted as $\tilde{u}_{\alpha} \cdot \mathcal{C}(E)$ means the collection of all compact convex subsets of $E$. Then clearly, $\tilde{u} \in \mathcal{F}(E)$ means $\tilde{u}_{\alpha} \in \mathcal{C}(E)$ for all $\alpha \in[0,1]$. Let $\mathbf{R}$ be the set of all real numbers and let $\mathcal{C}(\mathbf{R})$ be the set of all bounded closed intervals in $\mathbf{R}$. The elements of $\mathcal{F}(\mathbf{R})$ are called fuzzy numbers. The addition and the scalar multiplication on $\mathcal{F}(\mathbf{R})$ are well-known. See Puri and Ralescu(1985) for the details. The following results are known, so the proofs are omitted.

Lemma 1.1. (Chen-wei Xu(1992), Kurano et al.(1998)).

(i) For any $\tilde{m}, \tilde{n} \in \mathcal{F}(\mathbf{R})$ and $\lambda \geq 0$, it holds that $\tilde{m}+\tilde{n} \in \mathcal{F}(\mathbf{R})$ and $\lambda \tilde{m} \in \mathcal{F}(\mathbf{R})$.

(ii) Let $E_{1}$ and $E_{2}$ be convex compact subsets. If $\tilde{u} \in \mathcal{F}\left(E_{1}\right)$ and $\tilde{p} \in \mathcal{F}\left(E_{1} \times E_{2}\right)$ satisfy $\tilde{p}(x, \cdot) \in \mathcal{F}\left(E_{2}\right)$ for $x \in E_{1}$, then $\sup _{x \in E_{1}}\{\tilde{u}(x) \wedge \tilde{p}(x, \cdot)\} \in \mathcal{F}\left(E_{2}\right)$,

where $a \wedge b=\min \{a, b\}$ for real numbers $a, b$.

Now we will formulate the dynamic fuzzy system.

Definition 1.2. (Kurano et al.(1992)). The pair of $(S, \tilde{q})$ is called a dynamic fuzzy system if the following conditions (i) and (ii) are satisfied:

(i) The state space $S$ is a convex compact subset of some Banach space. In general, the state of the system is simply called as a fuzzy state and it is denoted by an element of $\mathcal{F}(S)$.

(ii) The law of motion for the system is based on a time-invariant fuzzy relations $\tilde{q}: S \times S \mapsto[0,1]$, and we assume $\tilde{q} \in \mathcal{F}(S \times S)$ and $\tilde{q}(x, \cdot) \in \mathcal{F}(S)$ for $x \in S$.

If the dynamic fuzzy system $(S, \tilde{q})$ is given, then we consider a sequential transition of states as follows. Firstly, a fuzzy state $\tilde{s} \in \mathcal{F}(S)$ is moved to a new fuzzy state $Q(\tilde{s})$ after a unit time has passed, where $Q: \mathcal{F}(S) \mapsto \mathcal{F}(S)$ is defined by

$$
Q(\tilde{s})(y):=\sup _{x \in S}\{\tilde{s}(x) \wedge \tilde{q}(x, y)\} \quad \text { for } y \in S .
$$


Note that the map $Q$ is well-defined by Lemma 1.1. Explicitly, for the dynamic fuzzy system $(S, \tilde{q})$ with a given initial fuzzy state $\tilde{s} \in \mathcal{F}(S)$, a sequence of fuzzy states $\left\{\tilde{s}_{t}\right\}_{t=1}^{\infty}$ is defined by

$$
\tilde{s}_{1}:=\tilde{s} \quad \text { and } \quad \tilde{s}_{t+1}:=Q\left(\tilde{s}_{t}\right) \quad(t \geq 1) .
$$

We need the following preliminaries to define fuzzy stopping times for this sequence $\left\{\tilde{s}_{t}\right\}_{t=1}^{\infty}$, which are given in the next section. Associated with the fuzzy relation $\tilde{q}$, the corresponding maps $Q_{\alpha}: \mathcal{C}(S) \mapsto \mathcal{C}(S)(\alpha \in[0,1])$ are defined as follows: For $D \in \mathcal{C}(S)$,

$$
Q_{\alpha}(D):= \begin{cases}\{y \in S \mid \tilde{q}(x, y) \geq \alpha \text { for some } x \in D\} & \text { if } \alpha>0 \\ \operatorname{cl}\{y \in S \mid \tilde{q}(x, y)>0 \text { for some } x \in D\} & \text { if } \alpha=0\end{cases}
$$

where $\mathrm{cl}$ means the closure of a set. From the assumption on $\tilde{q}$, the maps $Q_{\alpha}$ are welldefined. The iterates $Q_{\alpha}^{t}(t \geq 0)$ are defined by setting $Q_{\alpha}^{0}:=I$ (identity) and iteratively,

$$
Q_{\alpha}^{t+1}:=Q_{\alpha} Q_{\alpha}^{t} \quad(t \geq 0)
$$

In the following lemma, which is easily verified by the idea in the proof of Lemma 1 of Kurano et al.(1992), the $\alpha$-cuts of fuzzy state, $Q(\tilde{s})_{\alpha}$, are specified using $Q_{\alpha}$ of (1.3).

Lemma 1.3. (Kurano et al.(1992,1996)). For any $\alpha \in[0,1]$ and $\tilde{s} \in \mathcal{F}(S)$, we have:

(i) $Q(\tilde{s})_{\alpha}=Q_{\alpha}\left(\tilde{s}_{\alpha}\right)$;

(ii) $\tilde{s}_{t, \alpha}=Q_{\alpha}^{t-1}\left(\tilde{s}_{\alpha}\right) \quad(t \geq 1)$,

where $\tilde{s}_{\alpha}$ and $\tilde{s}_{t, \alpha}$ are the $\alpha$-cuts of fuzzy state $\tilde{s}$ and $\tilde{s}_{t}$ respectively and $\left\{\tilde{s}_{t}\right\}_{t=1}^{\infty}$ is defined by (1.2) with the initial state $\tilde{s}_{1}=\tilde{s}$.

\section{Fuzzy stopping times}

In this section, we define a fuzzy stopping time to be discussed here, and we introduce a new class of fuzzy stopping times, which is constructed through subsets of $\alpha$-cuts of fuzzy states. For the sake of simplicity, denote $\mathcal{F}:=\mathcal{F}(S)$ and let $\mathcal{F}^{\prime}$ be a subset of $\mathcal{F}$.

Definition 2.1. (Kurano et al.(1998)). A fuzzy stopping time on $\mathcal{F}^{\prime}$ is a fuzzy relation $\tilde{\sigma}: \mathcal{F}^{\prime} \times\{1,2, \cdots\} \mapsto[0,1]$ such that, for each fuzzy state $\tilde{s} \in \mathcal{F}^{\prime}, \tilde{\sigma}(\tilde{s}, t)$ is non-increasing in $t$ and there exists a natural number $t(\tilde{s}) \geq 1$ with $\tilde{\sigma}(\tilde{s}, t)=0$ for all $t \geq t(\tilde{s})$.

We note here that ' $\tilde{\sigma}=0$ ' represents 'stop' and ' $\tilde{\sigma}=1$ ' represents 'continuity' in the grade of membership (Kurano et al.(1998)). Between the two decisions, the intermediate value ' $0<\tilde{\sigma}<1$ ' is a notion of 'fuzzy stopping'. A fuzzy stopping time $\tilde{\sigma}(\tilde{s}, t)$ means the degree of 'continuity' at time $t$ starting from a fuzzy state $\tilde{s}$. The set of all fuzzy stopping times on $\mathcal{F}^{\prime}$ is denoted by $\Sigma\left(\mathcal{F}^{\prime}\right)$. 
Definition 2.2. A fuzzy stopping time $\tilde{\sigma} \in \Sigma\left(\mathcal{F}^{\prime}\right)$ is called monotone if there exists a map $\delta: \mathcal{F}^{\prime} \mapsto[0,1]$ satisfying

(i) $\delta(Q(\tilde{s})) \leq \delta(\tilde{s})$, and

(ii) $\tilde{\sigma}(\tilde{s}, t)=\bigwedge_{l=1}^{t} \delta\left(\tilde{s}_{l}\right)$ for all $\tilde{s} \in \mathcal{F}^{\prime}$ and $t \geq 1$,

where $\left\{\tilde{s}_{t}\right\}_{t=1}^{\infty}$ is defined by (1.2) with $\tilde{s}_{1}=\tilde{s}$.

The real-valued map $\delta$ is called a support of $\tilde{\sigma}$. The definition means a natural and good property for fuzzy stopping times, which is simple and easy to calculate optimal stopping times in actual optimization problems. The degree of monotone fuzzy stopping times is given by only the fuzzy state at current time $t$. Therefore, in stopping problems, the criterion is reduced whether the fuzzy state at current time $t$ belongs to the optimal stopping region or not.

We now construct a class of monotone fuzzy stopping times. For the purpose of this construction, we assume the following conditions.

Condition C1. For each $\alpha \in[0,1]$, there exists a non-empty subset $\mathcal{K}_{\alpha}$ of $\mathcal{C}(S)$ satisfying

$$
Q_{\alpha}\left(\mathcal{K}_{\alpha}\right) \subset \mathcal{K}_{\alpha}
$$

Using this subset $\mathcal{K}_{\alpha}$, we define a sequence of subsets $\left\{\mathcal{K}_{\alpha}^{t}\right\}_{t=1}^{\infty}$ inductively by

$$
\begin{array}{ll}
\mathcal{K}_{\alpha}^{1} & :=\mathcal{K}_{\alpha} ; \\
\mathcal{K}_{\alpha}^{t} & :=\left\{c \in \mathcal{C}(S) \mid Q_{\alpha}(c) \in \mathcal{K}_{\alpha}^{t-1}\right\} \quad(t \geq 2) .
\end{array}
$$

Clearly, $\mathcal{K}_{\alpha}^{t}=Q_{\alpha}^{-1}\left(\mathcal{K}_{\alpha}^{t-1}\right)=Q_{\alpha}^{-(t-1)}\left(\mathcal{K}_{\alpha}\right)$. Also, it holds from (2.1) that $\mathcal{K}_{\alpha}^{t} \subset \mathcal{K}_{\alpha}^{t+1}(t \geq$ 1). To simplify our discussion, we assume the following condition holds henceforth.

Condition C2. For all $\alpha \in[0,1]$, it holds that

$$
\mathcal{C}(S)=\bigcup_{t=1}^{\infty} \mathcal{K}_{\alpha}^{t} .
$$

For $c \in \mathcal{C}(S)$ and $\alpha \in[0,1]$, we define a stopping time $\hat{\sigma}_{\alpha}(c)$ by

$$
\hat{\sigma}_{\alpha}(c):=\min \left\{t \geq 1 \mid c \in \mathcal{K}_{\alpha}^{t}\right\} .
$$

This is the first entry time of a closed interval $c(\in \mathcal{C}(S))$ with the grade $\alpha$. We define a restricted class $\hat{\mathcal{F}}(\subset \mathcal{F})$ by

$$
\hat{\mathcal{F}}:=\left\{\tilde{s} \in \mathcal{F} \mid \hat{\sigma}_{\alpha}\left(\tilde{s}_{\alpha}\right) \text { is non-increasing in } \alpha \in[0,1]\right\} .
$$

Using the class $\left\{\hat{\sigma}_{\alpha}\left(\tilde{s}_{\alpha}\right) \mid \alpha \in[0,1]\right\}$, for the restricted element $\tilde{s} \in \hat{\mathcal{F}}$, we define

$$
\hat{\sigma}(\tilde{s}, t):=\sup _{\alpha \in[0,1]}\left\{\alpha \wedge 1_{D_{\alpha}}(t)\right\} \quad(t \geq 1),
$$


where $1_{D_{\alpha}}$ is the indicator of a set $D_{\alpha}:=\left\{t \geq 1 \mid \hat{\sigma}_{\alpha}\left(\tilde{s}_{\alpha}\right)>t\right\}$. This is the usual technique to construct a fuzzy number from a family of level sets. Then we obtain the following theorem.

Theorem 2.3. Under Conditions C1 and C2, the following (i) and (ii) hold.

(i) $\hat{\sigma}_{\alpha}\left(\tilde{s}_{\alpha}\right)=\min \{t \geq 1 \mid \hat{\sigma}(\tilde{s}, t)<\alpha\} \quad$ for $\tilde{s} \in \hat{\mathcal{F}}$ and $\alpha \in[0,1]$.

(ii) $\hat{\sigma}=\hat{\sigma}(\tilde{s}, t)(\tilde{s} \in \hat{\mathcal{F}}, t \geq 1)$ of (2.6) is a fuzzy stopping time on $\hat{\mathcal{F}}$.

Proof. By the definition, $\hat{\sigma}(\tilde{s}, t)<\alpha$ is equivalent to $\hat{\sigma}_{\alpha}\left(\tilde{s}_{\alpha}\right) \leq t$. This fact shows (i). From Condition C2, there exists $t^{*} \geq 1$ with $\tilde{s}_{0} \in \mathcal{K}_{0}^{t^{*}}$. So, $\hat{\sigma}_{\alpha}\left(\tilde{s}_{\alpha}\right) \leq \hat{\sigma}_{0}\left(\tilde{s}_{0}\right) \leq t^{*}$ for all $\alpha \in[0,1]$, which implies that $\hat{\sigma}(\tilde{s}, t)=0$ for all $t \geq t^{*}$ by the definition of $\hat{\mathcal{F}}$. Since $\hat{\sigma}(\tilde{s}, t+1) \leq \hat{\sigma}(\tilde{s}, t)$ holds clearly for $t \geq 1$ from (2.6), we also obtain (ii).

In order to show the monotone property of $\hat{\sigma}$, we need the following lemma.

Lemma 2.4. Let $\tilde{s} \in \hat{\mathcal{F}}$. Then

(i) $\hat{\sigma}(\tilde{s}, t)=\alpha$ if and only if, for any $\epsilon>0, \tilde{s}_{\alpha+\epsilon} \in \mathcal{K}_{\alpha+\epsilon}^{t}$ and $\tilde{s}_{\alpha-\epsilon} \notin \mathcal{K}_{\alpha-\epsilon}^{t}$;

(ii) $\tilde{s}_{t} \in \hat{\mathcal{F}} \quad(t \geq 1)$.

Proof. By (2.6), $\hat{\sigma}(\tilde{s}, t)=\sup \left\{\alpha \mid \hat{\sigma}_{\alpha}\left(\tilde{s}_{\alpha}\right)>t\right\}$. So, (i) follows from (2.4). From Lemma 1.3(ii), for $l \geq 1, \hat{\sigma}_{\alpha}\left(\left(\tilde{s}_{l}\right)_{\alpha}\right)=\hat{\sigma}_{\alpha}\left(\tilde{s}_{l, \alpha}\right)=\hat{\sigma}_{\alpha}\left(Q_{\alpha}^{l-1}\left(\tilde{s}_{\alpha}\right)\right)$. By $(2.2)$ and $(2.4)$,

$$
\begin{aligned}
\hat{\sigma}_{\alpha}\left(\left(\tilde{s}_{l}\right)_{\alpha}\right) & =\min \left\{t \geq 1 \mid Q_{\alpha}^{l-1}\left(\tilde{s}_{\alpha}\right) \in \mathcal{K}_{\alpha}^{t}\right\} \\
& =\min \left\{t \geq 1 \mid \tilde{s}_{\alpha} \in \mathcal{K}_{\alpha}^{t+l-1}\right\} \\
& =\max \left\{\hat{\sigma}_{\alpha}\left(\tilde{s}_{\alpha}\right)-(l-1), 1\right\},
\end{aligned}
$$

and it is non-increasing in $\alpha \in[0,1]$ since $\tilde{\boldsymbol{s}} \in \hat{\mathcal{F}}$. Therefore we obtain (ii).

Theorem 2.5. Let $\tilde{\boldsymbol{s}} \in \hat{\mathcal{F}}$ be given and assume that Conditions $C 1$ and C2 hold. Then, $\hat{\sigma}=\hat{\sigma}(\tilde{s}, t), t \geq 1$, is a monotone fuzzy stopping time with the initial state $\tilde{s}$.

PROOF. Let $\left\{\tilde{s}_{t}\right\}_{t=1}^{\infty}$ be defined by $(1.2)$ with $\tilde{s}_{1}=\tilde{s}$. First, we will prove that

$$
\hat{\sigma}(\tilde{s}, t+r)=\hat{\sigma}(\tilde{s}, t) \wedge \hat{\sigma}\left(\tilde{s}_{t+1}, r\right) \text { for } t, r \geq 1 .
$$

Note that $\hat{\sigma}\left(\tilde{s}_{t+1}, r\right)$ is well-defined from Lemma 2.4(ii). Let $\alpha=\hat{\sigma}(\tilde{s}, t+r)$. From Lemma 2.4(i), we have

$$
\tilde{s}_{\alpha+\epsilon} \in \mathcal{K}_{\alpha+\epsilon}^{t+r} \quad \text { and } \quad \tilde{s}_{\alpha-\epsilon} \notin \mathcal{K}_{\alpha-\epsilon}^{t+r} \quad \text { for any } \epsilon>0 .
$$

Noting $Q_{\alpha}^{t}\left(\mathcal{K}_{\alpha}^{l}\right)=\mathcal{K}_{\alpha}^{l-t}(1 \leq t<l)$ and Lemma $1.3($ ii), we obtain

$$
\tilde{s}_{t+1, \alpha+\epsilon}=Q_{\alpha+\epsilon}^{t}\left(\tilde{s}_{\alpha+\epsilon}\right) \in Q_{\alpha+\epsilon}^{t}\left(\mathcal{K}_{\alpha+\epsilon}^{t+r}\right)=\mathcal{K}_{\alpha+\epsilon}^{r}
$$


and

$$
\tilde{s}_{t+1, \alpha-\epsilon}=Q_{\alpha-\epsilon}^{t}\left(\tilde{s}_{\alpha-\epsilon}\right) \notin Q_{\alpha-\epsilon}^{t}\left(\mathcal{K}_{\alpha-\epsilon}^{t+r}\right)=\mathcal{K}_{\alpha-\epsilon}^{r} .
$$

Therefore, we get $\hat{\sigma}\left(\tilde{s}_{t+1}, r\right)=\alpha$ from Lemma 2.4(i). Namely, $\hat{\sigma}(\tilde{s}, t+r)=\hat{\sigma}\left(\tilde{s}_{t+1}, r\right)$. Since $\hat{\sigma}(\tilde{s}, t+r) \leq \hat{\sigma}(\tilde{s}, t)$ from Theorem $2.3\left(\right.$ ii), we obtain $\hat{\sigma}(\tilde{s}, t) \wedge \hat{\sigma}\left(\tilde{s}_{t+1}, r\right)=\alpha$, and so $(2.7)$ holds. Next, we put $\delta(\tilde{s})=\hat{\sigma}(\tilde{s}, 1)$ for $\tilde{s} \in \hat{\mathcal{F}}$. From $(2.7)$, we get

$$
\begin{aligned}
\hat{\sigma}(\tilde{s}, t) & =\hat{\sigma}(\tilde{s}, 1) \wedge \hat{\sigma}\left(\tilde{s}_{2}, t-1\right) \\
& =\hat{\sigma}(\tilde{s}, 1) \wedge \hat{\sigma}\left(\tilde{s}_{2}, 1\right) \wedge \hat{\sigma}\left(\tilde{s}_{3}, t-2\right) \\
& =\cdots \\
& =\bigwedge_{l=1}^{t} \hat{\sigma}\left(\tilde{s}_{l}, 1\right) \\
& =\bigwedge_{l=1}^{t} \delta\left(\tilde{s}_{l}\right) \quad \text { for } t \geq 1 .
\end{aligned}
$$

Since we also have $\delta(Q(\tilde{s})) \leq \delta(\tilde{s})$ from Theorem 2.3(ii), $\hat{\sigma}$ is a monotone fuzzy stopping time with $\tilde{s}$. The proof of this theorem is completed.

\section{Fuzzy stopping problems}

In this section, applying the results in the previous section, we obtain the optimal fuzzy stopping time for a fuzzy dynamic system with fuzzy rewards (see Kurano et al.(1996)) when the weighting function is additive.

Firstly, we formulate the stopping problem to be considered here. Let $\tilde{r}: S \times \mathbf{R} \mapsto$ $[0,1]$ be a fuzzy relation satisfying $\tilde{r} \in \mathcal{F}(S \times \mathbf{R})$ and $\tilde{r}(x, \cdot) \in \mathcal{F}(\mathbf{R})$ for $x \in S$. If the system is in a current fuzzy state $\tilde{\boldsymbol{s}} \in \mathcal{F}$, a fuzzy reward is earned:

$$
R(\tilde{s})(z):=\sup _{x \in S}\{\tilde{s}(x) \wedge \tilde{r}(x, z)\}, \quad z \in \mathbf{R} .
$$

Then we can define a sequence of fuzzy rewards $\left\{R\left(\tilde{s}_{t}\right)\right\}_{t=1}^{\infty}$, where $\left\{\tilde{s}_{t}\right\}_{t=1}^{\infty}$ is defined in $(1.2)$ with the initial fuzzy state $\tilde{s}_{1}=\tilde{s}$. Let

$$
\varphi(\tilde{s}, t):=\sum_{l=1}^{t-1} R\left(\tilde{s}_{l}\right) \quad \text { for } t \geq 1
$$

Note that (3.1) designates the summation of fuzzy numbers. For details, refer to Puri and Ralescu(1985) and Kurano et al.(1996). We need the following lemma, which is proved in Kurano et al. $(1992,1996)$.

Lemma 3.1. (Kurano et al.(1992,1996)). For $t \geq 1$ and $\alpha \geq 0$,

$$
\varphi(\tilde{s}, t)_{\alpha}=\sum_{l=1}^{t-1} R_{\alpha}\left(\tilde{s}_{l, \alpha}\right)
$$


holds, where

$$
R_{\alpha}\left(\tilde{s}_{l, \alpha}\right):= \begin{cases}\left\{z \in \mathbf{R} \mid \tilde{r}(x, z) \geq \alpha \text { for some } x \in \tilde{s}_{l, \alpha}\right\} & \text { if } \alpha>0 \\ \operatorname{cl}\left\{z \in \mathbf{R} \mid \tilde{r}(x, z)>0 \text { for some } x \in \tilde{s}_{l, \alpha}\right\} & \text { if } \alpha=0\end{cases}
$$

Let $g: C(\mathbf{R}) \mapsto \mathbf{R}$ be any additive map, that is,

$$
g\left[c^{\prime}+c^{\prime \prime}\right]=g\left[c^{\prime}\right]+g\left[c^{\prime \prime}\right] \text { for } c^{\prime}, c^{\prime \prime} \in C(S) .
$$

Adapting this map $g=g[\cdot]$ for a weighting function (see Fortemps and Roubens(1996)), for a fuzzy stopping time $\hat{\sigma} \in \Sigma(\hat{\mathcal{F}})$ and an initial fuzzy state $\tilde{s} \in \hat{\mathcal{F}}$, the scalarization of the total fuzzy reward is given by

$$
\begin{aligned}
G(\tilde{s}, \hat{\sigma}) & :=\int_{0}^{1} g\left[\varphi\left(\tilde{s}, \hat{\sigma}_{\alpha}\right)\right] d \alpha \\
& =\int_{0}^{1} g\left[\sum_{t=1}^{\hat{\sigma}_{\alpha}-1} R_{\alpha}\left(\tilde{s}_{t, \alpha}\right)\right] d \alpha
\end{aligned}
$$

where $\hat{\sigma}_{\alpha}$ means $\hat{\sigma}(\tilde{s}, \cdot)_{\alpha}=\min \{t \geq 1 \mid \hat{\sigma}(\tilde{s}, t)<\alpha\}$ for simplicity. Since $\varphi\left(\tilde{s}, \hat{\sigma}_{\alpha}\right) \in C(\mathbf{R})$ and the map $\alpha \mapsto g\left[\varphi\left(\tilde{s}, \hat{\sigma}_{\alpha}\right)_{\alpha}\right]$ is left-continuous in $\alpha \in(0,1]$, therefore the right-hand integral of (3.4) is well-defined. For a given $\mathcal{F}^{\prime}(\subset \mathcal{F})$, our objective is to maximize (3.4) over all fuzzy stopping times $\hat{\sigma} \in \Sigma\left(\mathcal{F}^{\prime}\right)$ for each initial fuzzy state $\tilde{s} \in \mathcal{F}^{\prime}$.

Definition 3.2. A fuzzy stopping time $\hat{\sigma}^{*}$ with $\tilde{s} \in \mathcal{F}^{\prime}$ is called an $\tilde{s}$-optimal if

$$
G(\tilde{s}, \hat{\sigma}) \leq G\left(\tilde{s}, \hat{\sigma}^{*}\right) \text { for all } \hat{\sigma} \in \Sigma\left(\mathcal{F}^{\prime}\right) \text {. }
$$

If $\hat{\sigma}^{*}$ is $\tilde{s}$-optimal for all $\tilde{s} \in \mathcal{F}^{\prime}$, then $\hat{\sigma}^{*}$ is called optimal in $\mathcal{F}^{\prime}$.

Now we will seek an $\tilde{s}$-optimal or an optimal fuzzy stopping time by using the results in the previous sections. For each $\alpha \in[0,1]$, let

$$
\mathcal{K}_{\alpha}(g):=\left\{c \in C(S) \mid g\left[R_{\alpha}\left(Q_{\alpha}(c)\right)\right] \leq 0\right\} .
$$

Hence we need the following Assumptions A1 and A2, which are assumed to hold henceforth.

Assumption A1. (Closedness). For all $\alpha \in[0,1], Q_{\alpha}\left(\mathcal{K}_{\alpha}(g)\right) \subset \mathcal{K}_{\alpha}(g)$.

By (2.2), we define a sequence $\left\{\mathcal{K}_{\alpha}^{t}(g)\right\}_{t=1}^{\infty}$ by

$$
\mathcal{K}_{\alpha}^{t}(g):=Q_{\alpha}^{-(t-1)}\left(\mathcal{K}_{\alpha}(g)\right) \text { for } t \geq 1
$$

Assumption A2. For all $\alpha \in[0,1], C(S)=\bigcup_{t=1}^{\infty} \mathcal{K}_{\alpha}^{t}(g)$.

Using the sequence $\left\{\mathcal{K}_{\alpha}^{t}(g)\right\}_{t=1}^{\infty}$ given in $(3.6)$, we define $\hat{\sigma}_{\alpha}, \hat{\mathcal{F}}, \hat{\sigma}$ and $\hat{\sigma}(\tilde{s}, \cdot)_{\alpha}$ by $(2.4)-(2.6)$. Then, from Theorems 2.3 and $2.5, \hat{\sigma}$ is a monotone fuzzy stopping time on $\hat{\mathcal{F}}$. The following theorem will be proved by applying the idea of the monotone policy (Chow et al.(1971), Kadota et al.(1996) and Ross(1970)) for stochastic stopping problems. 
TheOREM 3.3. Under Assumptions $A 1$ and A2, $\hat{\sigma}$ is an optimal monotone fuzzy stopping time in $\hat{\mathcal{F}}$.

ProOF. Firstly, we will consider a deterministic stopping problem which maximizes the reward of a weighting function $g\left[\varphi(\tilde{s}, t)_{\alpha}\right]$ over $t \geq 1$. Since $g$ is additive, $g\left[\varphi(\tilde{s}, t)_{\alpha}\right]=$ $\sum_{l=1}^{t-1} g\left[R_{\alpha}\left(\tilde{s}_{l, \alpha}\right)\right]$ holds. Therefore $g\left[\varphi(\tilde{s}, t)_{\alpha}\right] \geq g\left[\varphi(\tilde{s}, t+1)_{\alpha}\right]$ if and only if $\tilde{s}_{t, \alpha} \in K_{\alpha}(g)$. By Assumption A1, $\tilde{s}_{t, \alpha} \in K_{\alpha}(g)$ implies $g\left[\varphi(\tilde{s}, t)_{\alpha}\right] \geq g\left[\varphi(\tilde{s}, l)_{\alpha}\right]$ for all $l \geq t$. Together with (3.5), we obtain

$$
\left.\left.g[\varphi(\tilde{s}, \hat{\sigma}(\tilde{s}, \cdot)))_{\alpha}\right] \geq g[\varphi(\tilde{s}, \tilde{\sigma}(\tilde{s}, \cdot)))_{\alpha}\right]
$$

for all $\tilde{\sigma} \in \Sigma\left(\mathcal{F}^{\prime}\right)$ and $\alpha \in[0,1]$. This implies that $G(\tilde{s}, \hat{\sigma}) \geq G(\tilde{s}, \tilde{\sigma})$ for all $\tilde{\sigma} \in \Sigma\left(\mathcal{F}^{\prime}\right)$ by using (3.4). This completes the proof.

\section{A numerical example}

An example is given to illustrate the previous results of fuzzy stopping problem in this section. Let $S:=[0,1]$. The fuzzy relation $\tilde{q}$ and the fuzzy reward $\tilde{r}$ are given by

$$
\tilde{q}(x, y)=\left\{\begin{array}{ll}
1 & \text { if } y=\beta x \\
0 & \text { otherwise }
\end{array} \quad \text { and } \quad \tilde{r}(x, z)= \begin{cases}1 & \text { if } z=x-\lambda \\
0 & \text { otherwise }\end{cases}\right.
$$

for $x, y \in[0,1]$ and $z \in \mathbf{R}$, where $\lambda>0$ is an observation cost and $0<\beta<1$. Then, $Q_{\alpha}$ and $R_{\alpha}$ defined by (1.3) and (3.2) are independent of $\alpha$ and are calculated as follows:

$$
Q_{\alpha}([a, b])=[\beta a, \beta b] \quad \text { and } \quad R_{\alpha}([a, b])=[a-\lambda, b-\lambda]
$$

for $0 \leq a \leq b \leq 1$. is given as

Let $g[[a, b]]:=(a+2 b) / 3$ for $0 \leq a \leq b \leq 1$, which is additive. Then, $\mathcal{K}_{\alpha}:=\mathcal{K}_{\alpha}(g)$

$$
\mathcal{K}_{\alpha}=\{[a, b] \in C(S) \mid a+2 b \leq 3 \lambda / \beta\}
$$

So $\mathcal{K}_{\alpha}^{t}=Q_{\alpha}^{-(t-1)}\left(\mathcal{K}_{\alpha}\right)=\left\{[a, b] \in C(S) \mid a+2 b \leq 3 \lambda / \beta^{t}\right\}$. Since $\mathcal{K}_{\alpha}^{t}$ is independent of $\alpha$, we see that $Q_{\alpha}\left(\mathcal{K}_{\alpha}\right)=\left\{\beta[a, b] \mid[a, b] \in \mathcal{K}_{\alpha}\right\}$ and $\bigcup_{t=1}^{\infty} \mathcal{K}_{\alpha}^{t}=C(S)$. Thus Assumptions $\mathrm{A} 1$ and $\mathrm{A} 2$ in Section 3 are fulfilled in this example.

Let the initial fuzzy state be

$$
\tilde{s}(x):=\max \{1-4|2 x-1|, 0\} \quad \text { for } x \in[0,1] .
$$

For the stopping time $\hat{\sigma}_{\alpha}\left(\tilde{s}_{\alpha}\right)$ given in $(2.4)$, we easily obtain that $\tilde{s}_{\alpha}=[(3+\alpha) / 8,(5-$ $\alpha) / 8]$ and $\hat{\sigma}_{\alpha}\left(\tilde{s}_{\alpha}\right)=\min \left\{t \geq 1 \mid 13-\alpha \leq 24 \lambda \beta^{-t}\right\}$. Thus, as $\hat{\sigma}_{\alpha}\left(\tilde{s}_{\alpha}\right)$ is non-increasing in $\alpha \in[0,1]$, we have $\tilde{s} \in \hat{\mathcal{F}}$. Since $\hat{\sigma}_{\alpha}\left(\tilde{s}_{\alpha}\right) \in \mathcal{K}^{t}(g)$ means $13-\alpha \leq 24 \lambda \beta^{-t}$, we obtain

$$
\hat{\sigma}(\tilde{s}, t)=\min \left\{1, \max \left\{13-24 \lambda \beta^{-t}, 0\right\}\right\} .
$$

The numerical value of $\hat{\sigma}$ is given in Table 1 .

Table 1: An $\tilde{s}$-optimal fuzzy stopping time $(\lambda=0.48, \beta=0.98)$.

\begin{tabular}{|c|cccccccc|}
\hline$t$ & 1 & 2 & 3 & 4 & 5 & 6 & 7 & $\cdots$ \\
\hline$\hat{\sigma}(\tilde{s}, t)$ & 1 & 1 & .7603 & .5108 & .2552 & .00 & .00 & $\cdots$ \\
\hline
\end{tabular}




\section{Acknowledgments}

The authors would like to thank anonymous referees for valuable comments and suggestions.

\section{References}

Chen-wei $\mathrm{Xu}(1992)$ : On convexity of fuzzy sets and fuzzy relations. Inform. Science, 59, 92-102.

Chow, Y.S., Robbins, H. and Siegmund, D.(1971): The theory of optimal stopping: Great expectations, Houghton Mifflin Company, New York.

Fortemps, P. and Roubens, M.(1996): Ranking and defuzzification methods based on area compensation. Fuzzy Sets and Systems, 82, 319-330.

Kacprzyk, J.(1977): Control of a non-fuzzy system in a fuzzy environment with fuzzy termination time. System Sciences, 3, 325-341.

Kacprzyk, J. and Esogbue, A.O.(1996): Fuzzy dynamic programming: Main developments and applications. Fuzzy Sets and Systems, 81, 31-48.

Kadota, Y., Kurano, M. and Yasuda, M.(1996): Utility-optimal stopping in a denumerable Markov chain. Bull. Infor. Cyber. Res. Ass. Stat. Sci. 28, 15-21.

Kurano, M., Yasuda, M., Nakagami, J. and Yoshida, Y.(1992): A limit theorem in some dynamic fuzzy systems. Fuzzy Sets and Systems, 51, 83-88.

Kurano, M., Yasuda, M., Nakagami, J. and Yoshida, Y.(1996): Markov-type fuzzy decision processes with a discounted reward on a closed interval. Europ. Jour. of Oper. Res., 92, 649-662.

Kurano, M., Yasuda, M., Nakagami, J. and Yoshida, Y.(1998): An approach to stopping problems of a dynamic fuzzy system. submitted.

Novák, V.(1989): Fuzzy sets and their applications. Adam Hilder, Bristol-Boston.

Puri, M.L. and Ralescu, D.A.(1985): The concept of normality for fuzzy random variables. Ann. Prob., 13, 1373-1379.

Ross, S.M.(1970): Applied Probability Models with Optimization Applications, HoldenDay.

Yoshida, Y.(1994): Markov chains with a transition possibility measure and fuzzy dynamic programming. Fuzzy Sets and Systems, 51, 39-57.

Received May 22, 1998

Revised October 29, 1998 